\section{Learning by handshake?}

SIR-We recently reported that long-term potentiation (LTP) in some hippocampal synapses is associated with a slow increase in the sensitivity of CA1 neurons to analogues of the neurotransmitter Lglutamate' ${ }^{\prime}$. This result implies that postsynaptic mechanisms can underlie the long-lasting enhancement of synaptic efficiency in this experimental model of learning. Because LTP is generated within seconds, yet the postsynaptic change takes about an hour to reach its maximum, we suggested that presynaptic mechanisms account for the early phase of LTP. On the basis of these data, and two other recent publications ${ }^{2.3}$, Stevens ${ }^{+}$suggested in his News and Views article a four-step mechanism for the generation of LTP (see his figure in ref. 4). Stevens proposes that a complex sequence of intercellular messengers provides a 'handshaking' mechanism whereby checks are instituted to ensure that the necessary conditions are met for having an appropriate increase in synaptic strength.

Although such a complex scheme is possible, we wish to point out that it is not necessary to account for the data ${ }^{1-3}$. An alternative explanation is that the postsynaptic calcium influx triggers the postsynaptic change and that the delay is inherent in the sequence of biochemical processes involved.

We believe that the increase in sensitivity involves protein kinases, because we find that the increase is blocked by the potent protein kinase $\mathrm{C}$ inhibitor $\mathrm{K}-252 \mathrm{~b}$ (unpublished observations). Because manipulations of the postsynaptic cell seem to block LTP from its onset, there still seems to be the need for a retrograde messenger.

Graham L. COLLINGRIDGe STEPHEN N. DAVIES

Department of Pharmacology,

School of Medical Sciences,

University of Bristol,

Bristol BS8 1TD, UK

ROBIN A. J. LESTER

Vollum Institute for Advanced Biomedical Research,

3181 S.W. Sam Jackson Park Road,

Portland, Oregon 97201, USA

KLAUS G. REYMANN

Institute of Neurobiology and Brain

Research,

Academy of Sciences GDR,

Leipzigstrasse 44,

DDR-3090,

Magdeburg, GDR

1. Davies, S.N., Lester, R.A.J., Reymann, K.G. \& Collingridge, G.L. Nature 338, 500-503 (1989).

2. Kaver, J.A., Malenka, R.C. \& Nicoll, R.A. Nature 334, 250 252 (1988)

3. Malinow, R., Madison, D.V. \& Tsien, R.W. Nature 335 821-824 (1988).

4. Stevens, C.F. Nature 338, 460-461 (1989).

Mystery of meteoritic fusion

SIR-Although the concentrations of radioisotopes generated by cosmic rays in recently fallen stony and iron meteorites are in agreement with expectation, it has been known for several years that tritium is exceptional in that its concentration in iron meteorites is deficient ${ }^{1}$.

The usual explanation is that tritium diffuses rapidly out of iron meteorites ${ }^{2.3}$, but this is contradicted by measurements of helium-3, a decay product of tritium. Both the concentration of helium- 3 and the helium-3/helium- 4 ratio indicate that tritium has been present throughout the radiation history of the meteorites in the amounts expected from known crosssections, and that it must decompose within the meteorites.

Some years ago, I put forward ${ }^{4.5}$ the radical alternative explanation that tritium in a metal matrix no longer decays with a 12-year but with a much shorter half-life. This suggestion has been vigorously criticized (see, for example, ref. 6), but the low content of tritium in iron meteorites remains a puzzle, at least to me.

The recent reports ${ }^{7.8}$ that deuterium fusion can occur rapidly in a metal matrix are also puzzling. Deuterium-deuterium fusion may either lead to tritium plus hydrogen or helium-3 plus a neutron, but relatively few neutrons have been observed and also little tritium. At first sight, this

\section{Radiation risk}

SIR-Dunster ${ }^{1}$ argues in favour of radiation protection standards based on detriment rather than risk. Ideally the setting of standards is a biphasic process; first, a level of risk or detriment deemed to be acceptable should be set, then the doses and exposures that equate to this risk should be derived. The first phase is a political judgement, the second a scientific interpretation of available data, in which scientific rigour has to be compromised by the need for utility.

The existing framework recommended by the International Commission of Radiation Protection (ICRP) ${ }^{2}$, and accepted in the United Kingdom, deems acceptable for radiation workers the risks associated with 'safe' industries. The process of defining the relationship between risk or detriment and dose is fraught with uncertainty. Dunster argues in favour of detriment on two main counts: first, projected years of life lost to attributable causes is more relevant than deaths; and second, latency defers the risk to later in life even to the point that it may not be expressed.

It is true that 'years of life lost' is less sensitive to risk projection models than is risk itself, but the factor 2 involved is less than the uncertainty involved in inferring risks using data from the best available would exclude both deuterium reactions.

I would like to suggest that the reaction leading to tritium is predominant, but that the isotope is detected in only small amounts because it decays rapidly, in the metal matrix, to helium-3. The experiment suggested as a test of the hypothesis that tritium decays rapidly in iron meteorites would also apply in the deuterium fusion experiments - to look for a rapid growth of helium-3.

I acknowledge that the suggestion that the half-life of tritium can be lowered in a metal matrix is contradicted by the wellestablished theory of $\beta$-decay, but the low concentration of tritium in iron meteorites and the small quantities of neutrons and tritium found in the deuterium fusion experiments are both so puzzling that the possibility that some unanticipated effect is affecting the decay constant should not be excluded.

MURRY A. TAMERS

Beta Analytic Inc.,

PO Box 248113, Coral Gables,

Florida 33124, USA

1. Arnold, J.R. et al. J. Geophys. Res. 66, 3, 519 (1961).

2. Fisher, D.E., J. Geophys. Res. 72, 1, 351 (1967).

3. Tilles, D. Nature 201808 (1964)

4. Tamers, M.A. Nature 197, 276 (1963)

5. Tamers, M.A. Nature 200,565 (1963)

6. Tilles, D. Nature 200, 563 (1963)

7. Fleischman, M. \& Pons, S. J. electroanalyt. Chem. 261 301-8 (1989)

8. Jones, S.E. et al. Nature 338, 737-740 (1989)

epidemiology ${ }^{3}$. Indeed the UNSCEAR report ${ }^{3}$ is at considerable pains to point out the uncertainty in these estimates. Add to this the uncertainty involved in extrapolating to low doses and low doserates (UNSCEAR ${ }^{3}$ proposes a value between 2 and 10) and a factor 2 becomes inconsequential. The ameliorating effect of taking latency into account is small.

The demands of utility, at least in the present system recommended by the ICRP ${ }^{2}$, also distort the scientific basis in several ways. For example, the risk of breast cancer induction by radiation is negligible except for women exposed under the age of $40-$ about a quarter of the population - and yet this risk is deemed to be averaged over the whole population.

Thus risk, in relation to radiationinduced cancer at low doses and doserates, is intrinsically very uncertain; the need to use it in a workable framework of protection standards inevitably devalues it further. In expressing the harmful effects of radiation, careful consideration should be given to the degree of refinement that it is legitimate to use.

\section{MRC Radiobiology Unit,}

\section{K.F. BAVERSTOCK}

\section{Chilton, Didcot, Oxon OX11 ORD, UK}

1. Dunster. H.J. Nature 337, 311 (1989).

2. ICRP Publ. 26 (Pergamon, Oxford, 1977

3. United Nations Scientific Committee on the Effects of Atomic Radiation: Sources and Effects of Ionizing Radiation (United Nations, New York, 1988). 